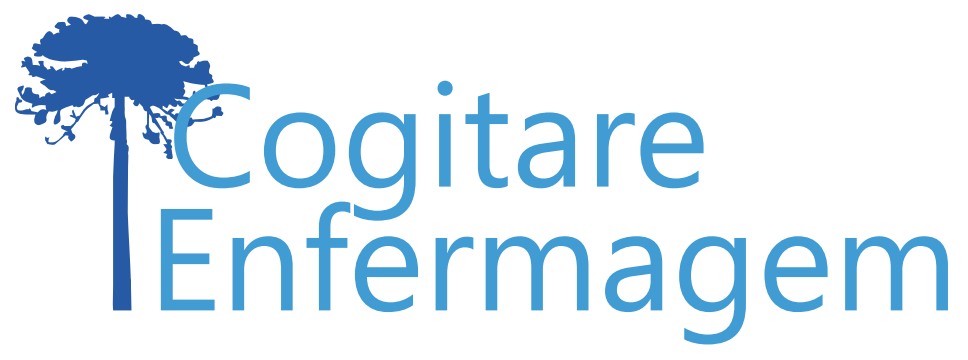

\title{
ESTRATIFICAÇÃO DE RISCO PARA TROMBOEMBOLISMO VENOSO EM PACIENTES DE UM HOSPITAL PÚBLICO DO DISTRITO FEDERAL
}

lara Gevila Lima da Silva1, Elaine Barros Ferreira², Priscila Roberta Silva Rocha

\begin{abstract}
RESUMO
Objetivo: estratificar o risco de pacientes clínicos para tromboembolismo venoso e características associadas em hospital público do Distrito Federal.

Método: estudo prospectivo (follow-up de 60 dias), entre 15 de agosto e 15 de outubro de 2016 com pacientes clínicos maiores de 18 anos e tempo de internação superior a 48 horas. Foram analisadas características demográficas, clínicas e profiláticas. Os sujeitos foram separados em dois grupos: alto risco (casos) e o baixo risco (controle) para tromboembolismo venoso, conforme o Escore de Pádua.

Resultados: foi elevada a prevalência de pacientes com alto risco e os fatores de risco mais frequentes foram: redução de mobilidade 59 (58,42\%), idade avançada 54 (53,47\%) e infecções ou doenças reumáticas 47 (46,53\%).

Conclusão: ressaltamos a importância da identificação das características de risco, além da atuação da equipe multiprofissional para otimizar a estratificação do risco, implementação de medidas profilaticas e terapêuticas precoces.
\end{abstract}

DESCRITORES: Tromboembolia; Tromboembolia venosa; Embolia Pulmonar; Fatores de Risco; Enfermagem; Equipe de Enfermagem.

COMO REFERENCIAR ESTE ARTIGO:

Lima I, Ferreira EB, Rocha PRS. Estratificação de risco para tromboembolismo venoso em pacientes de um hospital público do distrito federal. Cogitare enferm. [Internet]. 2019 [acesso em "colocar data de acesso, dia, mês abreviado e ano"]; 24. Disponível em: http://dx.doi.org/10.5380/ce.v24i0.56741.

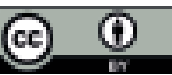

Este obra está licenciado com uma Licença Creative Commons Atribuição 4.0 Internacional.

${ }^{1}$ Enfermeira. Especialista em Saúde do Adulto e Idoso. Escola Superior De Ciências Da Saúde/ Fundação de Ensino e Pesquisa em Ciências da Saúde. Brasília, DF, Brasil. ()

${ }^{2}$ Enfermeira. Doutora em Enfermagem. Docente de Enfermagem da Universidade de Brasília. Brasília, DF, Brasil. 9

${ }^{3}$ Enfermeira. Doutora em Ciências da Saúde. Docente de Enfermagem da Universidade de Brasília. Brasília, DF, Brasil. $\odot$ 


\title{
STRATIFICATION OF RISK FOR VENOUS THROMBOEMBOLISM IN PATIENTS OF A PUBLIC HOSPITAL OF THE FEDERAL DISTRICT
}

\author{
ABSTRACT \\ Objective: to stratify the risk of venous thromboembolism and associated characteristics in \\ clinical patients of a public hospital of the Federal District. \\ Method: prospective study (60-day follow-up), between August 15 and October 15, 2016, with \\ clinical patients aged over 18 years hospitalized for more than 48 hours. The demographic, \\ clinical and prophylactic characteristics were analyzed. The subjects were divided into two \\ groups: high risk (cases) and low risk (control) for venous thromboembolism, according to the \\ Padua Score. \\ Results: there was a high prevalence of high-risk patients and the most frequent risk factors \\ were: reduced mobility 59 (58.42\%), advanced age 54 (53.47\%), and rheumatic infections or \\ diseases 47 (46.53\%). \\ Conclusion: we emphasize the importance of the identification of risk characteristics, as well as \\ the practice of the multidisciplinary team to optimize the risk stratification and to implement \\ early prophylaxis and therapeutic measures.
}

DESCRIPTORS: Thromboembolism; Venous thromboembolism; Pulmonary Embolism; Risk factors; Nursing; Nursing team.

\section{ESTRATIFICACIÓN DE RIESGO PARATROMBOEMBOLISMO VENOSO EN PACIENTES DE UN HOSPITAL PÚBLICO DE DISTRITO FEDERAL}

\begin{abstract}
RESUMEN:
Objetivo: estratificar el riesgo de pacientes clínicos para tromboembolismo venoso y identificar características asociadas en hospital público de Distrito Federal.

Método: estudio prospectivo (follow-up de 60 días), hecho entre 15 de agosto y 15 de octubre de 2016 con pacientes clínicos mayores de 18 años y tiempo de ingreso superior a 48 horas. Se analizaron las características demográficas, clínicas y profilácticas. Se organizaron los individuos en dos grupos: alto riesgo (casos) y bajo riesgo (control) para tromboembolismo venoso, de acuerdo a la Puntuación de Pádua.

Resultados: se elevó la prevalencia de pacientes con alto riesgo y los factores de riesgo más frecuentes fueron: reducción de movilidad 59 (58,42\%), edad avanzada $54(53,47 \%)$ e infecciones o enfermedades reumáticas 47 (46,53\%).

Conclusión: se resalta la importancia de la identificación de las características de riesgo, además de la actuación del equipo multiprofesional para optimizar la estratificación de riesgo, implementación de medidas profilácticas y terapéuticas precoces.
\end{abstract}

DESCRIPTORES: Tromboembolía; Tromboembolía venosa; Embolia Pulmonar; Factores de Riesgo; Enfermería; Equipo de Enfermería. 
O tromboembolismo venoso (TEV) é uma das principais causas de mortes evitáveis no ambiente intra-hospitalar, sendo frequentemente associado a complicações ${ }^{(1)}$. O TEV é uma doença vascular trombótica de etiologia multifatorial, resultante da formação de trombos no sistema venoso, por fatores idiopáticos ou desconhecidos, abrangendo duas alterações: a trombose venosa profunda (TVP) e a tromboembolia pulmonar (TEP)(2).

Os mecanismos envolvidos nesse processo foram descritos por Rudolf Virchow como a tríade de fatores trombogênicos, a saber: estase venosa, dano endotelial e hipercoagulação. A American College of Chest Physicians (ACCP) ${ }^{(3)}$ aponta o câncer ativo, história prévia de TEV, mobilidade reduzida, trombofilia conhecida, trauma ou cirurgia no último mês, idade avançada, insuficiência cardíaca ou respiratória, infecções e/ou doenças reumatológicas, infarto agudo do miocárdio ou acidente vascular cerebral, obesidade e terapia hormonal atual, como fatores de risco para TEV.

A ocorrência de trombofilias em pacientes não cirúrgicos está associada ao aumento dos custos hospitalares e maior tempo de internação, bem como ao aumento do risco de recidiva de TEV quando não anticoagulados adequadamente ${ }^{(4)}$.

O risco de morte precoce entre pacientes com TEP é 18 vezes maior em comparação com TVP isolada. Para quase um quarto dos pacientes com TEP, a apresentação clínica inicial é morte súbita(5). No Brasil, um registro incluindo mais de 27.000 pacientes cirúrgicos e clínicos, evidenciou a subutilização de profilaxia para TEV em $25 \%$ dos pacientes em alto risco e em $45 \%$ daqueles com risco moderado, predominando em pacientes não cirúrgicos ${ }^{(6)}$.

A estratificação do risco de TEV é um processo dinâmico, que objetiva adequar o método profilático e monitorar as terapêuticas ${ }^{(7-8)}$. Dentre as escalas de estratificação do risco para pacientes clínicos, a ACCP sugere o Escore de Pádua como forma de avaliação ${ }^{(3)}$. Este escore avalia os 14 fatores de risco descritos na Tabela 1. Cada fator pontuado é somado para gerar um risco cumulativo. A pontuação final define o nível de risco de TEV do paciente ${ }^{(9)}$, sendo escore $\geq 4$ alto risco e escore $<4$ baixo risco.

Tabela 1 - Avaliação de risco Escore de Pádua(3)

\begin{tabular}{ll} 
Características & Pontuação \\
\hline Câncer ativo & 3 \\
\hline TEV prévio & 3 \\
\hline Redução de mobilidade & 3 \\
\hline Trombofilia conhecida & 3 \\
\hline Trauma ou cirurgia recente & 2 \\
\hline Idade avançada & 1 \\
\hline ICC ou IR & 1 \\
\hline Infecções ou doenças reumáticas & 1 \\
\hline IAM ou AVC & 1 \\
\hline Obesidade & 1 \\
\hline Terapia hormonal & 1
\end{tabular}

Escore $\geq 4$ - alto risco e escore $<4$ - baixo risco.

Fonte: American College of Chest Physicians (ACCP) ${ }^{(3)}$ 
Ensaios clínicos ${ }^{(10-11)}$ evidenciam a segurança, a efetividade e baixo custo da tromboprofilaxia em pacientes hospitalizados com risco de TEV. Os métodos profiláticos podem ser mecânicos e farmacológicos, podendo ser usados independentemente ou em associação(3).

Contudo, há diversas barreiras na aplicabilidade da tromboprofilaxia, dentre elas o desconhecimento dos profissionais acerca das diretrizes atuais, dificuldades socioeconômicas, resistência às mudanças de práticas e ausência de protocolos, bem como o receio de sangramentos ${ }^{(10)}$.

É necessário que haja profissionais capacitados para reconhecer e estratificar os riscos para TEV o mais precocemente possível, haja vista que o tempo de início da profilaxia interfere em uma maior exposição a complicações e mortalidade por TEP e TVP(9).

Este estudo teve como objetivo estratificar o risco de pacientes clínicos para TEV (Escore de Pádua) de um hospital público do Distrito Federal (DF) e verificar as características associadas ao escore de risco.

\section{MÉTODO}

Trata-se de estudo observacional, prospectivo (follow-up de 60 dias), realizado no período de 15 de agosto a 15 de outubro de 2016 com pacientes internados em uma Unidade de Clínica Médica (UCM) de um hospital público terciário da Secretaria do Estado de Saúde do Distrito Federal (SES/DF).

Foram incluídos indivíduos com idade $\geq 18$ anos e internação $\geq 48 \mathrm{~h}$, foram excluídos pacientes que apresentaram dados incompletos. Durante o período do estudo, houve um total de 103 admissões, dois pacientes não foram incluídos devido ao período de internação ter sido menor do que 48 horas. Dessa forma, 101 pacientes compuseram a amostra deste estudo, conforme apresentado na Figura 1.

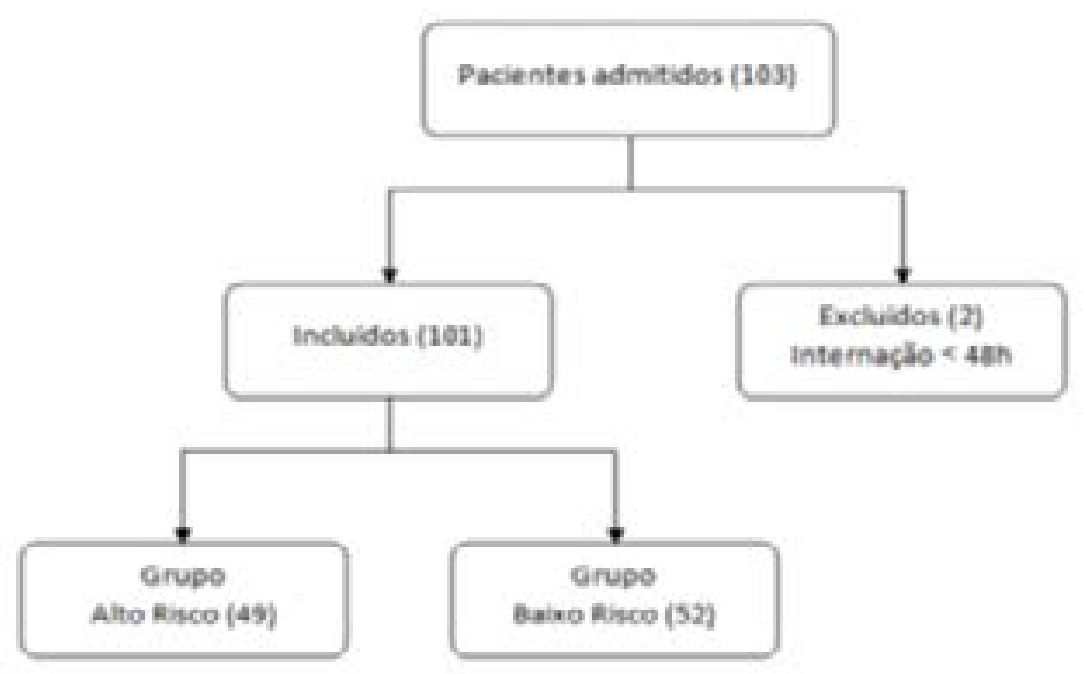

Figura 1 - Fluxo de informação da seleção da amostra. Brasília, DF, Brasil, 2016

O estudo foi composto por duas etapas: na primeira etapa, foram documentadas características sociodemográficas e clínicas dos pacientes, bem como a estratificação do risco para TEV de acordo com o Escore de Pádua( ${ }^{(3)}$. Na segunda etapa, os pacientes 
com Escore de Pádua $\geq 4$ (alto risco para TEV) foram acompanhados diariamente durante o período de internação, por meio do prontuário eletrônico, e o desfecho clínico foi documentado.

Para a coleta dos dados, foi utilizado instrumento construído pelas autoras, o qual reuniu dados de identificação do paciente (número de registro de prontuário eletrônico); variáveis sociodemográficas (idade e sexo); variáveis clínicas (diagnóstico de acordo com o CID, tempo de internação, fatores de risco para TEV, dados antropométricos); pontuação do Escore de Pádua (Tabela 1) e variáveis profiláticas utilizadas para TEV (tempo de uso e tipo de profilaxia utilizada). Os dados antropométricos para cálculo do IMC foram obtidos por meio de registros do serviço de nutrição (dados aferidos ou estimados na admissão, de acordo com a condição física do paciente), as demais variáveis analisadas eram obtidas através do prontuário eletrônico.

Os dados obtidos foram armazenados em uma base de dados gerida por um sistema gerenciador de banco de dados relacional, MySQL ${ }^{(12)}$. Posteriormente foram exportados para o programa Microsoft Office Excel, para classificação e agrupamento. A análise estatística foi realizada por meio do programa SAS (Analytics software \&solutions), versão University Edition para Windows.

As variáveis contínuas foram reportadas com medidas de tendência central, como média e desvio padrão, e as variáveis categóricas foram descritas por números absolutos e percentuais. Para verificação dos fatores associados ao alto risco de TEV, os sujeitos do estudo foram elencados em dois grupos: baixo risco de TEV (controle) e alto risco de TEV (casos), conforme a estratificação do Escore de Pádua. O teste Shapiro-Wilk foi usado para avaliar a normalidade dos dados. Para dados que assumiram distribuição não normal, utilizou-se o teste não paramétrico de Mann-Whitney. Para a comparação entre os grupos, utilizaram-se os testes Qui-quadrado e teste Exato de Fisher. Foi considerado significativo valor de $p<0,05$.

O presente obteve aprovação do Comitê de Ética em Pesquisa da Fundação de Ensino e Pesquisa em Ciências da Saúde (FEPECS) sob o parecer de número 1.655.708.

\section{RESULTADOS}

Dos 101 pacientes incluídos no estudo, a maioria $56(55,45 \%)$ era idosa, com predomínio de indivíduos do sexo masculino $58(57,43 \%)$ e com $50(49,5 \%)$ eutróficos. O tempo médio de internação foi de $12,12(+15,04)$ dias, os fatores de risco para TEV observados com maior frequência foram: redução de mobilidade $59(58,42 \%)$, idade avançada $54(53,47 \%)$ e infecções ou doenças reumáticas $47(46,53 \%)$ (Tabela 2). Segundo o Escore de Pádua, $52(51,48 \%)$ pacientes foram classificados como baixo risco (escore < 4 ) e $49(48,52 \%)$ com escore $\geq 4$, classificados como alto risco para o desenvolvimento de tromboembolismo venoso (Tabela 2).

Tabela 2 - Características demográficas e clínicas e o alto risco para desenvolvimento de TEV entre os pacientes internados na UCM de um hospital público do DF. Brasília, DF, 2016 (continua)

\begin{tabular}{lcc} 
Características demográficas e clínicas & \multicolumn{2}{c}{ Total de pacientes } \\
\cline { 2 - 3 } & $\mathbf{N}(101)$ & $\%$ \\
\hline Idade (anos) (média \pm DP) & $61,95 \pm 18,31$ & - \\
\hline Classes de idade & & \\
\hline$\leq 59$ anos & 45 & 44,55 \\
\hline$\geq 60$ anos & 56 & 55,45 \\
\hline
\end{tabular}




\begin{tabular}{lcc}
\hline Sexo masculino & 58 & 57,43 \\
\hline IMC (média \pm DP) & $23,05 \pm 4,79$ & - \\
\hline Tempo de internação (dias) (média \pm DP) & $12,12 \pm 15,04$ & - \\
\hline Escore de Pádua & & \\
\hline Baixo risco $(<4)$ & 52 & 51,58 \\
\hline Alto risco $(\geq 4)$ & 49 & 48,42 \\
\hline Fatores de risco* & & \\
\hline Câncer ativo & 12 & 11,88 \\
\hline TEV prévio & 4 & 3,96 \\
\hline Redução de mobilidade & 59 & 58,42 \\
\hline Trombofilia conhecida & 8 & 7,92 \\
\hline Trauma ou cirurgia recente & 7 & 6,93 \\
\hline Idade avançada & 54 & 53,47 \\
\hline ICC ou IR & 36 & 35,64 \\
\hline Infecções ou doenças reumáticas & 47 & 46,53
\end{tabular}

ICC: insuficiência cardíaca; IAM: infarto agudo do miocárdio; AVC: acidente vascular cerebral; IR: insuficiência renal; DP: desvio padrão; IMC: índice de massa corporal

Dentre as comorbidades apresentadas pelos pacientes, observou-se a hipertensão arterial sistêmica (HAS) como a condição mais frequente $48(47,5 \%)$, seguida de infecções $39(38,6 \%)$ e diabetes $36(35,6 \%)$.

$\mathrm{Na}$ segunda parte do estudo (Tabela 3), a comparação dos grupos (casos e controles) evidenciou que os pacientes de alto risco ( $\geq 4$, segundo Escore de Pádua) eram significativamente mais velhos ( $\geq 60$ anos) $(p=0,0183)$. O sexo masculino e o IMC não apresentaram diferença estatística quando associados ao risco elevado de TEV. O grupo de alto risco apresentou mais fatores de risco para TEV $(p<, 0001)$. O tempo médio de internação do grupo alto risco foi maior que o grupo baixo risco $(13,4 \pm 15$ versus $10,92 \pm 14,98)$, porém não houve significância estatística $(p=0,3710)$.

Tabela 3 - Associação entre as características demográficas e clínicas e o alto risco para desenvolvimento de TEV entre os 101 pacientes internados na UCM de um hospital público do DF. Brasília, DF, 2016 (continua)

\section{Variável}

Total de pacientes $\mathbf{N}=101$
Controle (52)

Baixo Risco
P-valor

$0,0183^{a}$

Idade

\section{Alto Risco}

\begin{tabular}{ll}
\hline$\leq 59$ anos & 16 \\
\hline$\geq 60$ anos (idoso) & 33 \\
\hline Sexo masculino & 28 \\
\hline IMC
\end{tabular}




\begin{tabular}{|c|c|c|c|}
\hline Magreza $(<18.5)$ & 12 & 7 & \multirow{5}{*}{$0,4892^{b}$} \\
\hline Eutrofia ( $\geq 18.5-<24,9)$ & 23 & 27 & \\
\hline Sobrepeso $(\geq 25-<29,9)$ & 11 & 11 & \\
\hline Obesidade $(\geq 30-<39,9)$ & 1 & 4 & \\
\hline Não informado & 2 & 3 & \\
\hline Tempo de internação (dias), média \pm DP & $13,4 \pm 15$ & $10,92 \pm 14,98$ & $0,3710^{c}$ \\
\hline Fatores de risco TEV (média $\pm \mathrm{DP}$ ) & $3,59 \pm 0,88$ & $1,51 \pm 0,61$ & $<, 0001^{\circ}$ \\
\hline Profilaxia medicamentosa ou mecânica & 26 & 3 & $<, 0001^{\circ}$ \\
\hline \multicolumn{4}{|l|}{ Desfecho clínico } \\
\hline Alta & 27 & 42 & $0,0026^{b}$ \\
\hline Internados & 6 & 2 & \\
\hline UTI & 1 & 1 & \\
\hline Óbito & 15 & 7 & $0,0350^{b}$ \\
\hline
\end{tabular}

$a=$ teste qui-quadrado, $b=$ teste de Fischer, $c=$ teste Mann Whitney

Observou-se que, dos 49 pacientes do grupo alto risco, 26 (53,06\%) utilizaram a profilaxia, com diferença estatística entre os grupos $(p<0,0001)$. Entre os pacientes estudados, a maioria que evoluiu para alta médica hospitalar era do grupo baixo risco $(p=0,0026)$. O óbito foi significativo no grupo de alto risco $(p=0,0350)$.

\section{DISCUSSÃO}

O TEV é um evento muitas vezes silencioso, que não só impõe substancial carga socioeconômica aos sistemas de saúde, mas também é responsável por gerar situações debilitantes para a saúde, tais como aumento da morbimortalidade, incapacidade e diminuição da qualidade de vida(13). De acordo com evidências científicas, um em cada seis casos de tromboembolismo poderiam ser evitados ${ }^{(14)}$.

A avaliação do risco de TEV representa um importante indicador de qualidade dos serviços de saúde ${ }^{(15-16)}$. A padronização de modelos de estratificação de risco é um dos principais pontos que estimulam o cenário de pesquisa, visando a adequação da profilaxia centrada no indivíduo e a otimização entre o processo de estratificação e a implementação de medidas preventivas ${ }^{(14)}$.

Em estudo multicêntrico ${ }^{(17)}$, realizado em países africanos, observou-se que 353 $(62,3 \%)$ apresentavam alto risco para o desenvolvimento de TEV. A maior ocorrência de alto risco para TEV se repete em estudo realizado com 104 pacientes em um hospital da região sul do Brasil(16), no qual 46 (44,23\%) dos pacientes apresentavam alto risco e $4(3,85 \%)$ altíssimo risco para TEV. Conforme esses estudos citados, verifica-se que o alto risco para TEV é um fato da realidade hospitalar. E assim como demonstrado nesta pesquisa, apresenta alta incidência em pacientes clínicos. Uma possível explicação para semelhança entre os estudos é o perfil populacional e as comorbidades presentes, uma vez que os autores ${ }^{(16)}$ encontraram como fatores de risco a idade $>40$ anos $75(72,1 \%)$, e comorbidades, tais como insuficiência cardíaca congestiva e neoplasias.

Neste estudo, a idade média elevada também foi identificada como fator estatisticamente significante associado ao alto risco de TEV ( $p=0.0183)$. Essa característica 
é condizente com o cenário atual de pacientes clínicos, pelo aumento de internações por doenças crônicas degenerativas decorrentes do envelhecimento populacional(18-19). Em estudo de revisão sistemática ${ }^{(5)}$, verificou-se que as taxas de TEV aumentam acentuadamente com a idade, independente do sexo, sendo a taxa de incidência geral ajustada pela idade mais elevada para os homens $(1,2: 1)^{(5)}$. A associação entre obesidade e TEV ainda não foi totalmente elucidada.

Em estudo americano(20), a frequência de óbito no grupo de alto risco foi maior que no grupo baixo risco, dados estes concordantes com o presente estudo no qual a taxa de óbito no grupo alto risco foi duas vezes maior. A ACCP indica aos pacientes clínicos, estratificados com alto risco, a tromboprofilaxia anticoagulante farmacológica, e como medidas não farmacológicas preconizam-se: meias de compressão graduadas, intermitentes e dispositivos pneumáticos de compressão(3). Nesta pesquisa, $26(53,06 \%)$ pacientes de alto risco utilizaram a profilaxia medicamentosa, em consonância com outros estudos ${ }^{(6,20)}$.

Embora pesquisas multicêntricas referentes à classificação do risco estejam sendo realizadas, como ENDORS Estudy (Epidemiologic International Day for the Evaluationof Patientsat Risk for Venous Thromboembolism in the Acute Hospital Care Setting)(21), percebe-se subnotificação quanto à incidência de alto risco para TEV, principalmente em instituições que carecem de protocolos assistenciais específicos, o que aumenta a possibilidade de ocorrência de eventos tromboembólicos evitáveis, uma vez que a ausência de prescrição da profilaxia não é responsabilidade exclusiva do médico, mas sim de toda equipe multiprofissional envolvida ${ }^{(22-23)}$. É necessário buscar medidas que visem a melhora da frequência e da qualidade de utilização da tromboprofilaxia ${ }^{(24)}$.

Como fatores limitantes, este estudo evidenciou a inexistência de um protocolo institucional para a estratificação de risco de pacientes para o desenvolvimento de TEV, o que dificulta a identificação e o monitoramento da implementação das medidas profiláticas.

\section{CONCLUSÃO}

A frequência de pacientes clínicos com alto risco para TEV, segundo Escore de Pádua, foi elevada. Fatores de risco, como redução de mobilidade, idade avançada e infecções ou doenças reumáticas, apresentaram uma alta incidência na população estudada. A idade, a quantidade de fatores de risco, a utilização de profilaxia e o desfecho clínico mostraram associação estatística com o maior risco de desenvolver TEV.

Estudos como este auxiliam na identificação das principais características quanto ao perfil de risco para TEV de indivíduos em internação hospitalar, especialmente o paciente clínico, além de corroborar para a construção do conhecimento sobre a classificação de risco do tromboembolismo venoso em pacientes clínicos.

Ressalta-se que é necessária uma atuação multiprofissional que busque ferramentas para otimizar a estratificação do risco e prevenção ao TEV.

\section{REFERÊNCIAS}

1. Di Nisio M, Porreca E. Prevention of venous thromboembolism in hospitalized acutelyill medical patients: focus on the clinical utility of (low-dose) fondaparinux. Drug Des Devel Ther. [Internet]. 2013 [acesso em 10 dez 2016]; 2013(7). Disponível em: https://doi.org/10.2147/DDDT.S38042.

2. Saghazadeh A, Rezaei N. Inflammation as a cause of venous thromboembolism. Crit. Rev. Oncol. Hematol. [Internet]. 2016[acesso em 18 nov2016]; 99. Disponível em: https://doi.org/10.1016/j.critrevonc.2016.01.007.

3. Kahn SR, Lim WT, Dunn AS, Cushman M, Dentali F, Akl EA, et al. Prevention of VTE in non surgical 
patients: Antithrombotic Therapy and Prevention of Thrombosis, 9th ed: American College of Chest Physicians Evidence-Based Clinical Practice Guidelines. Chest. [Internet]. 2012 [acesso em 26 jan 2017]; 141(2). Disponível em: https://doi.org/10.1378/chest.11-2296.

4. Vedantham S, Piazza G, Sista AK, Goldenberg NA. Guidance for the use of thrombolytic therapy for the treatment of venous thromboembolism. J Thromb Thrombolysis. [Internet]. 2016 [acesso em 30 set 2016]; 41(1). Disponível em: https://doi.org/10.1007/s11239-015-1318-z.

5. Heit JA, Spencer FA, White RH. The epidemiology of venous thromboembolism. J Thromb Thrombolysis. [Internet]. 2016 [acesso em 02 jan 2017]; 41(1). Disponível em: https://doi.org/10.1007/s11239-015-1311-6.

6. Bastos M, Barreto SM, Caiafa JS, Rezende SM. Tromboprofilaxia: recomendações médicas e programas hospitalares. Rev. Assoc. Med. Bras. [Internet]. 2011 [acesso em 12 dez 2016]; 57(1). Disponível em: http:// dx.doi.org/10.1590/S0104-42302011000100022.

7. Khorana AA, Carrier M, Garcia DA, Lee AY. Guidance for the prevention and treatment of cancerassociated venous thromboembolism. J Thromb Thrombolysis. [Internet]. 2016 [acesso em 10 jan2017]; 41(1). Disponível em: https://doi.org/10.1007/s11239-015-1313-4.

8. Lenchus JD. Transitions in the Prophylaxis, Treatment and Care of Patients with Venous Thromboembolism. Adv Ther. [Internet]. 2016 [acesso em 19 dez 2016]; 33(1). Disponível em: https://doi.org/10.1007/s12325015-0271-8.

9. Liu X, Liu C, Chen X, Wu W, Lu G. Comparison between Caprini and Padua risk assessment models for hospitalized medical patients at risk for. Interact Cardiovasc Thorac Surg. [Internet]. 2016 [acesso em 15 dez 2016]; 23(4). Disponível em: https://doi.org/10.1093/icvts/ivw158.

10 Erzinger FL, Carneiro MB. How can prevention of venous thromboembolism be improved in a hospital with an oncological profile? J. vasc. bras. [Internet]. 2016 [acesso em 30 nov 2016]; 15(3). Disponível em: http://dx.doi.org/10.1590/1677-5449.003216.

11. Kahn SR, Morrison DR, Cohen JM, Emed J, Tagalakis V, Roussin A, et al. Interventions for implementation of thromboprophylaxis in hospitalized medical and surgical patients at risk for venous thromboembolism (review). Cochrane Database Syst Rev. [Internet] 2013 [acesso em 13 dez 2016]; 16(7). Disponível em: https://www.ncbi.nlm.nih.gov/pubmed/23861035.

12. Naramore E, Gerner J, Scouarnec YL, Stolz J, Michael KG. Beginning PHP 5, Apache, MySQL Web Development (Programmer to Programmer). Birmingham,UK: Wrox Press; 2005.

13. Tsai J, Grant AM, Beckman MG, Grosse SD, Yusuf HR, Richardson LC. Determinants of Venous Thromboembolism among Hospitalizations of US Adults: a multilevel analysis. PloS one. [Internet]. 2015 [acesso em 11 out 2016]; 10(4). Disponível em: https://doi.org/10.1371/journal.pone.0123842.

14. Nazarenko GI, Kleymenova EB, Payushik SA, Otdelenov VA, Sychev DA, Yashina LP. Decision support systems in clinical practice: The case of venous thromboembolism prevention. Int J Risk Saf Med. [Internet]. 2015 [acesso em 19 jan 2017]; 27(1). Disponível em: https://doi.org/10.3233/JRS-150709.

15. Joint Commission. [Internet]. 2015 [acesso em 12 dez 2016]. Disponível em: https://www.jointcommission. org/performance measurement.aspx.

16. Busato CR, Gomes RZ, Costa DMM, Zubiolo TFM. Evaluation of thromboprophylaxis in medium-sized general hospital. J. vasc. bras. [Internet]. 2014 [acesso em 29 out 2016]; 13(1). Disponível em: http://dx. doi. org/10.1590/jvb.2014.003.

17. Kingue S, Bakilo L, Mvuala R, Minkande JZ, Fifen I, Gureja YP, et al. Epidemiological African day for evaluation of patients at risk of venous thrombosis in acute hospital care settings. Cardiovasc J Afr. [Internet]. 2014 [acesso em 30 out 2016]; 25(4). Disponível em: https://doi.org/10.5830/CVJA-2014-025.

18. Kuchemann BA. Envelhecimento populacional, cuidado e cidadania: velhos dilemas e novos desafios. Soc. estado. [Internet]. 2012 [acesso em 17 dez 2016]; 27(1). Disponível em: http://dx.doi.org/10.1590/ S0102-69922012000100010. 
19. Heit JA, Ashrani AA, Crusan DJ, McBane RD, Petterson TM, Bailey KR. Reasons for the persistent incidence of venous thromboembolism. Thromb Haemost. [Internet]. 2017 [acesso em 18 dez 2016]; 117(2). Disponível em: http://dx.doi.org/10.1160/TH16-07-0509.

20. Vazquez F, Watman R, Tabares A, Gumpel C, Baldessari E, AB V. Risk of venous thromboembolic disease and adequacy of prophylaxis in hospitalized patients in Argentina: a multicentric cross-sectional study. Thromb J. [Internet]. 2014 [acesso em 10 nov 2016]; 12(15). Disponível em: https://doi.org/10.1186/14779560-12-15.

21. Cohen A, Tapson V, Bergmann J, Goldhaber S, Kakkar. Investigators E. Venous thromboembolism risk and prophylaxis in the acute care setting (ENDORSE study): a multinational cross-sectional study. Lancet. [Internet]. 2008 [acesso em 26 nov 2016]; 371. Disponível em: http://dx.doi.org/10.1016/S01406736(08)60202-0.

22. Fanikos J, Rao A, Seger AC, Carter D, Piazza G, Goldhaber SZ. Hospital Costs of Acute Pulmonary Embolism. Am J Med. [Internet]. 2013 [acesso em 13 jan 2017]; 126(2). Disponível em: http://dx.doi. org/10.1016/j.amjmed.2012.07.025.

23. Vitor SKS, Daou JP, Góis AFT. Prevenção de tromboembolismo (trombose venosa profunda e embolia pulmonar) em pacientes clínicos e cirúrgicos. Diagn tratamento. [Internet]. 2016 [acesso em 20 out 2016]; 21(2). Disponível em: http://files.bvs.br/upload/S/1413-9979/2016/v21n2/a5583.pdf.

24. Rocha ATC, Paiva EF, Araujo DM, Cardoso DN, Pereira ACH, Lopes AA, et al. Impact Of A Program For Venous Thromboembolism Prophylaxis In Hospitalized Patients In Four Hospitals In Salvador. Rev. Assoc. Med. Bras. [Internet]. 2010 [acesso em 20 set 2016]; 56(2). Disponível em: http://dx.doi.org/10.1590/S010442302010000200019.

Recebido: 05/12/2017

Finalizado: 08/03/2019

Autor Correspondente:

Priscila Roberta Silva Rocha

Universidade de Brasília

Centro Metropolitano, conjunto A, lote 01 - 72220-275 - Brasília, DF, Brasil

E-mail: priscillarocha@unb.br

Contribuição dos autores:

Contribuições substanciais para a concepção ou desenho do estudo; ou a aquisição, análise ou interpretação de dados do estudo - PRSR

Elaboração e revisão crítica do conteúdo intelectual do estudo - EBF

Aprovação da versão final do estudo a ser publicado - PRSR

Responsável por todos os aspectos do estudo, assegurando as questões de precisão ou integridade de qualquer parte do estudo - IL 